\title{
The passive and active contractile properties of the neurogenic, underactive bladder
}

\author{
John S. Young, Louise Johnston*, Camille Soubrane ${ }^{\dagger}$, Karen D. McCloskey*, \\ Gordon McMurray ${ }^{\dagger}$, Rachel Eccles ${ }^{\dagger}$ and Christopher H. Fry
}

Institute of Biosciences and Medicine, University of Surrey, Guildford, *Centre for Cancer Research and Cell Biology, School of Medicine, Dentistry and Biomedical Sciences, Queen's University Belfast, Belfast, ${ }^{\dagger}$ Pfizer Global Research and Development, Sandwich, Kent, UK

\section{What's known on the subject? and What does the study add?}

- Detrusor underactivity is highly prevalent, particularly in the elderly. It is assumed to result from detrusor failure, although detrusor contractility is often derived from urodynamics studies. Given that detrusor pressure and force are not proportional and urodynamics cannot identify the basis of the pathology, we produced a neurogenic animal model with a highly-compliant bladder and studied detrusor muscle properties, aiming to increase our understanding of the underlying pathology.

- Highly compliant bladders were characterized by reduced passive wall stiffness and stretched detrusor muscle strips exhibited an enhanced rate of relaxation. These detrusor strips displayed spontaneous contractions that were of greater amplitude (expressed as a ratio of bladder wall stiffness) than those of strips from sham-operated animals; spontaneous contractions increased in amplitude when stimulated by an agonist. These data imply that compliance is not the result of a reduction of detrusor contractility; we hypothesize that altered matrix properties reduce the magnitude with which force can be generated to void the bladder.

\section{Objective}

- To characterize passive and active changes in detrusor activity in a highly compliant bladder.

\section{Materials and Methods}

- Bladders from adult female Sprague-Dawley rats were used 5 weeks after lower thoracic (T8) spinal cord transection or a sham-operation.

- Passive wall properties were assessed by pressure-volume relationships from whole bladders and the tensile response of bladder strips after a rapid $(<0.5 \mathrm{~s})$ stretch.

- Active properties were assessed from the frequency and amplitude of spontaneous contractions of bladder strips, and their response to the inotropic TRPV4 agonist GSK1016790A.

\section{Results}

- Passive bladder wall stiffness of SCT bladders was significantly reduced compared to that of the sham-operated control group ( $N=6$ and 8 , respectively) and SCT bladder strips relaxed more quickly than those from sham-operated rats.

- The frequency of spontaneous contractions was reduced in SCT rats, and their amplitude, expressed as a ratio of bladder wall stiffness, was greater than in sham-operated rats.

- GSK1016790A (0.1 $\mu \mathrm{M})$ significantly increased amplitude in strips from both sham-operated and SCT groups.

\section{Conclusions}

- There is no evidence of contractile failure in a highly-compliant bladder. The observations of reduced passive bladder wall stiffness and an enhanced rate of stress relaxation lead to the conclusion that increased compliance is marked by altered matrix properties that dissipate muscle force, thereby generating low pressures.

- Contractile agonists may be effective for improving bladder function in detrusor underactivity.

\section{Keywords}

bladder, compliance, detrusor contractility, detrusor underactivity, spinal cord injury 


\section{Introduction}

Detrusor underactivity (DU) is a condition diagnosed by pressure-flow studies and is defined as a bladder contraction of either reduced strength and/or insufficient duration to complete voiding within a normal time-span [1]. Often, but not exclusively, it occurs in elderly patients and may be associated with overactive bladder symptoms [2]. DU is regarded primarily as failure of detrusor contractility, although this may require re-evaluation: DU is associated more with a reduced rate of contraction development rather than contractile strength, at least initially [3]; cholinergic agonists are not effective in improving an underactive bladder $[4,5]$ and there is no evidence for age-related impairment of detrusor contractility [6].

Urodynamic assessment of bladder contractility utilizes procedures during voiding or when the bladder is isovolumetric. Voiding measures include determination of the Watts factor [7,8], a bladder contractility index [9] or peak detrusor pressure measured at (or independent of) peak flow. Isovolumetric indices include the projected isovolumetric pressure from pressure-flow measurements [10] and maximum pressure during outflow tract occlusion [11]. Although informative, these methods are empirical and their usefulness varies between different patient groups $[10,12]$. Furthermore, urodynamics cannot yield fundamental information about the basis of DU (i.e. if it is a true decline of muscle contractility or whether some other factor is involved), which is compounded by the fact that detrusor pressure is not proportional to true detrusor force.

The present study aimed to develop a rat model of spinal cord transection (SCT) to generate a highly compliant bladder that develops low intraluminal pressures, aiming to characterize any changes in detrusor contractility (i.e. isometric force per unit cross-section of tissue). In a complementary study [13], we showed that such bladders developed smooth muscle hypertrophy, a loss of nerve endings and significant disruption of interstitial cell number in both the detrusor and lamina propria layers. The hypothesis that this was accompanied by reduced muscle contractility was tested.

\section{Materials and Methods}

\section{Experimental Groups}

Adult female Sprague-Dawley rats were used 5 weeks after SCT (animal weight 217-274 g) or a sham operation (animal weight 252-292 g). In some experiments, an unoperated group was also used (animal weight 260-300 g); all groups were age-matched. To produce SCT, rats were anaesthetized with isoflurane $(2 \%$ in $\mathrm{O} 2$,
$0.6 \mathrm{~L} / \mathrm{min}$ ), a laminectomy was performed at T8 and the spinal cord was sectioned with iris scissors. Complete sectioning was confirmed visually by slight retraction of the two spinal cord segments. To assist rats with their bladder voiding, a gentle abdominal compression was performed twice daily until restoration of the micturition reflex ( $\approx 2$ weeks) and they were injected daily, for the first week after surgery, with gentamicin $(2 \mathrm{mg} / \mathrm{kg})$. Procedures were carried out in accordance with the European Community Council Directive 86/609/EEC. The sham operation used the same procedure, although omitting the laminectomy.

Some 5 weeks after the procedure, rats were weighed, sacrificed by cervical dislocation and exsanguination, and the urinary bladder was removed and weighed. Bladders were transported in Ca-free Krebs solution (mM: $\mathrm{NaCl}$ 114, $\mathrm{KCl} 4.7, \mathrm{MgSO} 4$ 1.2, KH2PO4 1.2, NaHCO3 25, glucose $11.7 ; 95 \% \mathrm{O} 2 / 5 \% \mathrm{CO} 2, \mathrm{pH} 7.35)$ at $4{ }^{\circ} \mathrm{C}$ to the laboratory.

\section{Assessment of Wall Tension}

Whole bladders were catheterized and ligated around the urethra and bladder neck. Intravesical pressure and volume were monitored at $10 \mathrm{~Hz}$ (Spike 2; Cambridge Electronic Design, Cambridge, UK) and exported to Excel (Microsoft Corp., Redmond, CA, USA) for analysis. Bladders were filled (Minipuls3; Gilson, Middleton, WI, USA) at $12 \mathrm{~mL} / \mathrm{h}$ with Krebs until the pressure tended to a maximum or where leakage around the catheter was observed. Pressure-volume relationships were transformed into tension-length (i.e. wall tension vs bladder circumference) relationships, as described previously [14].

Isometric Tension Recording

In $\mathrm{Ca} 2+$-containing $(2.5 \mathrm{mM} \mathrm{CaCl} 2)$ Krebs solution, the urinary bladder was opened longitudinally from the bladder neck (posterior) to the top of the dome (anterior) and the trigone was removed. The mucosa was also removed from the remaining tissue by careful dissection using iris scissors; care was taken not to stretch or damage the underlying detrusor muscle. A single longitudinal strip $(\approx 5 \times 1 \mathrm{~mm})$ was mounted in a $5-\mathrm{mL}$ vertical organ bath under a tension of $10 \mathrm{mN}$, and isometric spontaneous contractions were measured. Preparations were continuously washed with fresh, gassed Krebs and allowed to equilibrate for $90 \mathrm{~min}$ before each experiment, with resting tension maintained at $10 \mathrm{mN}$. When the resting tension was stable, three sets of data were recorded: (i) a 5 -min period of spontaneous contractions; (ii) the tensile response after a rapid $(<0.5 \mathrm{~s})$ stretch to achieve an immediate further increase of resting tension by $10 \mathrm{mN}$; and (iii) upon relaxation of this additional tension, a 
15-min recording of spontaneous contractions in the presence of a contractile agonist GSK1016790A $(0.1 \mu \mathrm{M})$ [15] or its vehicle, dimethyl-sulphoxide $(0.1 \mathrm{mM})$. Data were acquired using an in-house manufactured analogue-digital converter attached to a computer running Notocord-hem software, version 4.2 (Notocord, Croissy-sur-Seine, France). At the end of each experiment, the wet weight $(W)$ and length $(l)$ of each strip were recorded to estimate cross-sectional area (CSA) using the formula: $\mathrm{CSA}=W / l \rho$, where $\rho$ is the density of tissue $(1.05 \mathrm{~g} / \mathrm{cm} 3)$.

\section{Analysis of Isometric Contraction Traces}

To analyze spontaneous contraction amplitude and basal tone, data were imported into Chart, version 5.5

(ADInstruments, Chalgrove, $\mathrm{UK}$ ) at $10 \mathrm{~Hz}$ and values were derived using a custom-written macro. Spontaneous contraction amplitude was the baseline-to-peak amplitude, and the baseline was the minimum isometric tension value, obtained as the mean over a 5 -min period. The frequency of spontaneous contractions was calculated using custom-written software (R. J. Young, Department of Physics, University of Lancaster, UK) by first performing a fast-Fourier transform on the data and then identifying the dominant frequency $\left(f_{0}\right)$ based on a Lorentzian fit to an amplitude vs frequency plot. Frequency values were not reported when the level of activity was so infrequent, or its frequency so variable, that a frequency profile could not accurately be described. Contraction data were integrated using Kaleidagraph, version 3.5 (Synergy Software, Reading, PA, USA) for 5-min period before and after intervention. The baseline was taken as mean of the 100 lowest values for each of these periods.

\section{Statistical Analysis}

Many data sets were not normally distributed and/or had different variances, as tested by Kolmogorov-Smirnov and Levene's tests, respectively. Data are therefore presented as median values with $25 \%(Q 1)$ and $75 \%(Q 3)$ interquartiles. Values from the sham-operated group are provided first. Differences between data sets were examined by a Wilcoxon rank test for two data sets or a Kruskal-Wallis test for multiple sets. $P<0.05$ was considered statistically significant. The variability of data within a given set was quantified by calculation of the coefficient of quartile deviation $(Q 3-Q 1) /(Q 3+Q 1)$. $N$ refers to the number of rats. Rates of relaxation for detrusor strips were calculated over a 2.5 -min period after retensioning (to $10 \mathrm{mN}$ ) using a single-order exponential decay fit with a non-zero asymptote (GraphPad Prism, version 4; GraphPad Software Inc., San Diego, CA, USA) and noting the decay constant, $k(s-1)$.

\section{Results \\ Ex Vivo Bladder Stress-Strain Properties}

The passive wall tension-length relationship obtained from ex vivo filling pressure-volume curves showed that bladders from SCT rats were less stiff than those from sham-operated rats. Wall tension $(T)$ vs bladder circumference ( $l$ ) curves (Fig. 1) were biphasic; the initial phase, characterized by a shallow slope, corresponded to filling of the bladder when the wall was under minimal tension. Once the bladder had filled and was more or less spherical, the $T / l$ relationship was linear. The slope of this linear phase was used to estimate passive bladder wall stiffness, $\Delta T / \Delta l$. The mean (range) stiffness of SCT bladders was significantly less compared to that of the sham-operated control group: $5.60(2.81-7.70)$ vs 1.61 $(0.92-2.04) \mathrm{mN} / \mathrm{mm} 2(N=6$ and 8 , respectively). As a result of the reduction in wall stiffness, SCT animal bladders were classified as highly-compliant.

\section{Spontaneous Contractile Activity}

Bladder strips from sham-operated and SCT rats generated spontaneous contractions (Fig. 2A). These were characterized to determine whether tissue from highly-compliant bladders had a phenotype of long-duration, fused contractions as a result of SCT [16]. A Fast-Fourier transform of tension-time data identified the dominant frequency $\left(f_{0}\right)$ of spontaneous contractions based

Fig. 1 Ex vivo wall tension ( $T$ ) vs bladder circumference (I) curves from sham-operated or spinal-cord transected (SCT) rats.

$\Delta$ tension, $\mathrm{mN} \cdot \mathrm{mm}^{-1}$

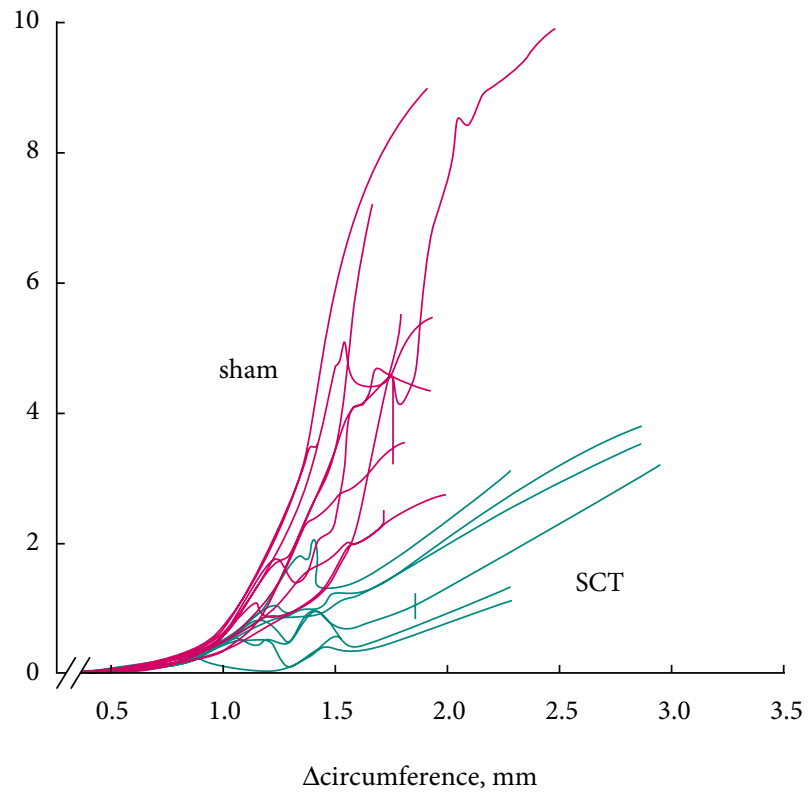


Fig. 2 Spontaneous contractions in detrusor strips from sham-operated and spinal-cord transected (SCT) rats. A, Spontaneous contraction recordings. B, Fourier transforms of the data showing the occurrence (power) of contractions at different frequencies, with dominant frequency $\left(f_{0}\right)$ indicated by arrows.

A

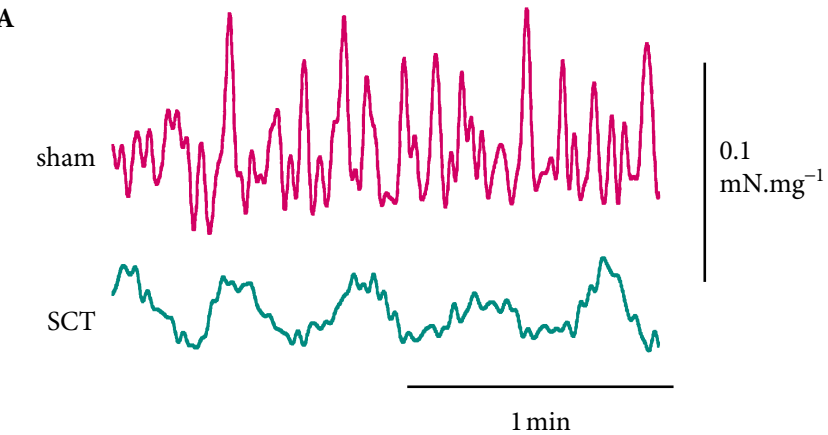

B

power, a.u.
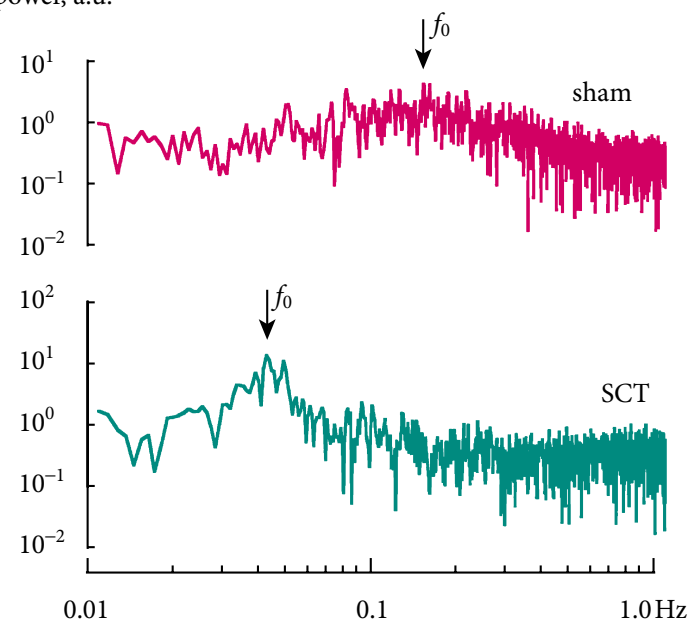

on a Lorentzian fit (Fig. 2B). The mean (range) frequency was significantly reduced in detrusor strips from SCT compared to sham-operated rats: $0.113(0.084-0.142)$ vs $0.051(0.038-0.068) \mathrm{Hz}(N=18$ and 18 , respectively) $(P<$ 0.001 ). The mean (range) amplitude of spontaneous contractions was not different between those from SCT and sham-operated rats: $4.8(2.3-6.5)$ vs $2.1(1.7-3.4) \mathrm{mN} / \mathrm{mm} 2$ ( $N=18$ and 18, respectively) (Fig. 3A). Although the SCT median value was less than for the sham-operated group, it did not reach statistical significance. In addition, the variability of amplitudes was not different between the two groups, as indicated by the mean (range) coefficient of quartile deviation: $0.96(0.53-1.37)$ vs $0.47(0.27-0.65)$.

The reduced passive stiffness of SCT bladder strips will reduce the magnitude of a recorded isometric contraction for the same contractile effort made by detrusor muscle. This is because the force developed by the muscle would be dissipated by the less stiff passive mechanical properties of
Fig. 3 Magnitude of spontaneous contractions in detrusor strips from sham-operated or spinal-cord transected (SCT) rats. A, Absolute tension values. B, Values normalized to passive stiffness. Median values (25\%, 75\% interquartiles). ${ }^{*} P<0.05$ Wilcoxon rank test vs sham-operated values.
A

tension, $\mathrm{mN} \cdot \mathrm{mm}^{-2}$

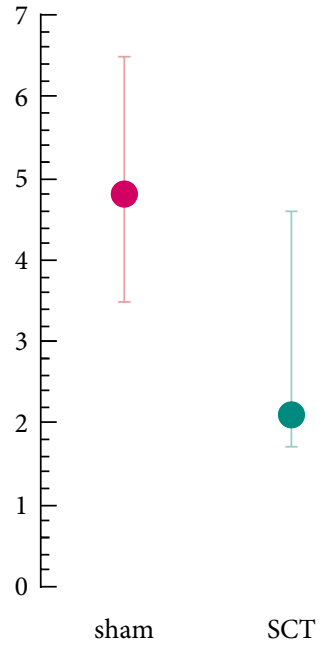

B

normalised tension

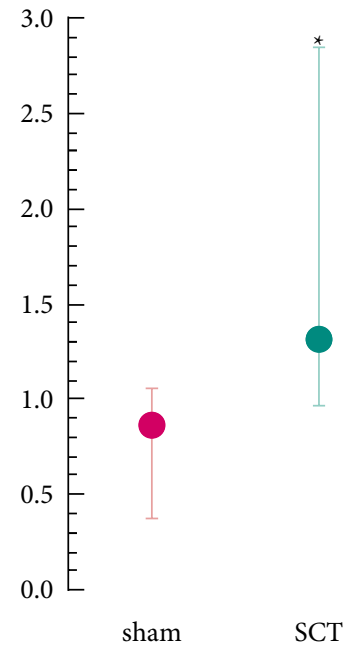

the tissue. To correct for this effect, the amplitudes of spontaneous contractions $(\mathrm{mN} / \mathrm{mm} 2)$ were expressed as a ratio of bladder wall stiffness $(\mathrm{mN} / \mathrm{mm} 2)$ from which the strip was dissected, yielding a dimensionless value of tension independent of wall stiffness (Fig. 3B). With this transformation, the mean (range) amplitude of spontaneous contractions was significantly greater in SCT rats compared to sham-operated rats: $1.31(0.97-2.85)$ vs $0.86(0.38-1.05)$ $\mathrm{mN} / \mathrm{mm} 2(P<0.001)$.

\section{Passive Stress-Relaxation Properties}

Detrusor strips were rapidly stretched to increase resting tension by a further $10 \mathrm{mN}$ and then allowed to relax. Figure $4 \mathrm{~A}$ shows the decay of resting tension with superimposed spontaneous contractions. Strips from SCT rats relaxed significantly more quickly than those from sham-operated rats; the mean (range) decay constants, $k$, were $0.0034(0.0031-0.0061)$ vs $0.010(0.0081-0.020) / \mathrm{s}(N=$ 17 and 18, respectively); the asymptotic values were not different in the two groups (median of $47 \%$ and $43 \%$, respectively).

\section{Control Experiments}

Data from detrusor strips of an unoperated animal group were also collected to determine whether surgery itself altered the contractile phenotype and, accordingly, were compared with those from the sham-operated group. The 
Fig. 4 Stress-relaxation of detrusor strips from sham-operated or spinal-cord transected (SCT) rats. A, Traces of spontaneous contractions and relaxation of baseline tension. B, Values of rate constants of baseline relaxation. Median values (25\%, $75 \%$ interquartiles). ${ }^{*} P<0.05$ Wilcoxon rank test vs sham-operated values.
A

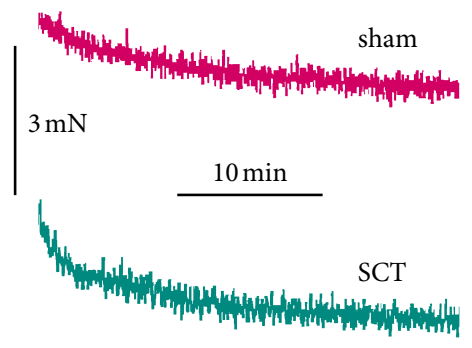

B

rate constant, $\mathrm{s}^{-1} \times 100$

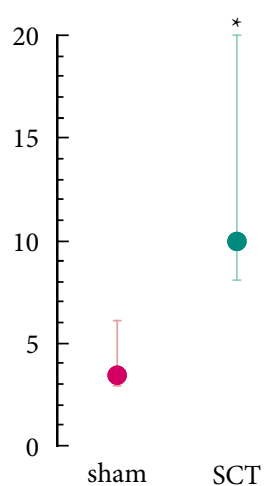

spontaneous contraction dominant frequency, $f_{0}(0.085[0.077-0.103] \mathrm{Hz}, N=24)$, amplitude $(3.2$ $[2.2-5.9] \mathrm{mN} / \mathrm{mg})$ and decay constant, $k(0.40$ [0.34-0.055] min-1, $N=18$ ) were not significantly different from sham-operated values and, in the case of $f_{0}$ and $k$ values, they were different from the SCT group.

\section{Response to a Detrusor-Selective Contractile Agonist}

GSK1016790A $(0.1 \mu \mathrm{M})$, a potent detrusor-selective contractile agonist [15], was tested on baseline tension and the amplitude of spontaneous contractile activity in sham-operated (Fig. 5A) and SCT detrusor strips (Fig. 5B). The effect of vehicle alone is also shown. Figure 5C summarizes the effect of GSK1016790A on contraction amplitude and shows that vehicle had no effect, although the drug significantly increased the amplitude in strips from both sham-operated and SCT groups. The percentage increase in the two groups was not significantly different.

\section{Discussion}

Detrusor underactivity is a prevalent condition that is estimated to occur in almost two-thirds of elderly incontinent nursing home patients [17]. Existing pharmacotherapy is ineffective, with symptoms being reported to worsen after anti-cholinergic therapy $[4,5]$. The condition is often misdiagnosed in men as bladder outlet obstruction $[4,18]$, such that $\alpha$-adrenoceptor antagonists may also worsen symptoms. To direct the development of effective therapies, it is important to determine whether detrusor underactivity is primarily a problem of contractile failure or an alternative aetiology.
Fig. 5 The effect of GSK101670A (0.1 $\mu \mathrm{M})$ on spontaneous contractions in detrusor strips from sham-operated or spinal-cord transected (SCT) rats. A, Recordings in vehicle or GSK101670A from sham-operated bladders. B, Recordings from SCT bladders. C, Summary of spontaneous contraction amplitude in vehicle or GSK101670A (pre-exposure = 100\%). Median values $(25 \%, 75 \%$ interquartiles). ${ }^{*} P<0.05$ Wilcoxon rank test GSK101670A vs vehicle.
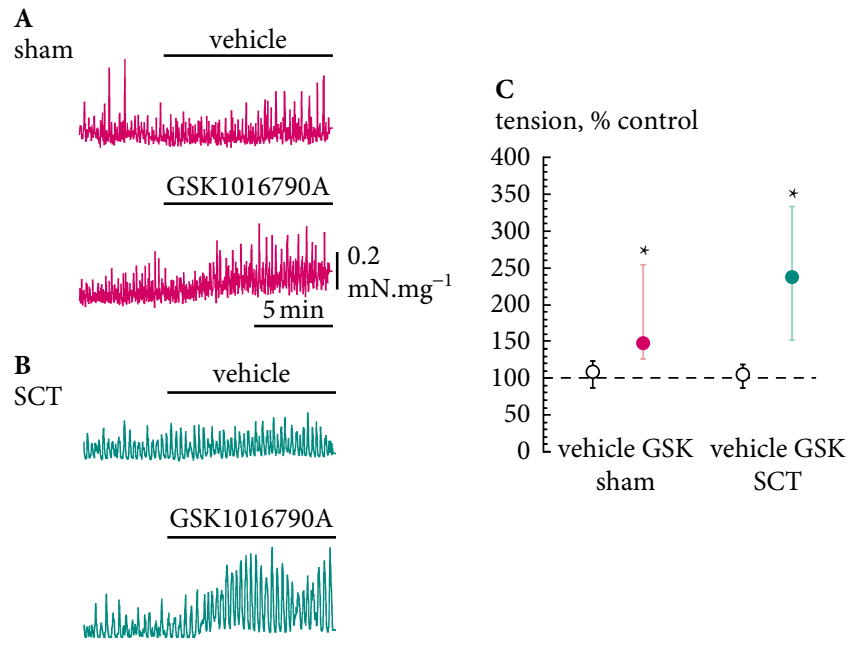

Highly-compliant bladders were generated by leaving rats for 5 weeks after lower thoracic (T8) SCT. At earlier times before surgery $(\approx 2$ weeks), the bladder is already hypertrophied but has not developed a hypercompliant phenotype observed after the longer interval in these experiments $[16,19]$. The principal objective was to determine whether the reduced intravesical pressures were accompanied by reduced detrusor contractility or were the result of another cause. A parallel study [13] recording the morphological features of these bladders showed significant denervation, which is characteristic of detrusor underactivity $[4,20,21]$, as well as a loss of interstitial cells between muscle bundles. An ex vivo preparation circumvented the possible impact of altered sensory function [22]; how this contributes to detrusor underactivity in vivo warrants further investigation.

\section{Contractile Performance of SCT Detrusor Muscle}

Detrusor strips exhibited spontaneous contractions of greater duration and lower frequency in SCT rats and were characteristic of those recorded in overactive bladder models [18]. However, their amplitude was not significantly different from those recorded in control strips. When the magnitude of the contraction was normalized to unit passive ex vivo stiffness, the magnitude of contractions was greater in the SCT group. The rationale for this normalization is that the tension generated by detrusor smooth muscle cells is transmitted through the extracellular matrix before being recorded by the force transducer. Thus, 
dissipation of muscular energy through a more compliant extracellular matrix will reduce the measured tension.

A significant rate of passive stress relaxation will also reduce the measured peak contractile force by acting as a viscous damper. An increased rate was measured in detrusor strips from SCT rats and would be expected to have a greater influence on these strips. A quantitative relationship has been described previously [23] and, using the above rate constants, would reduce the peak force of spontaneous contractions by $\approx 2 \%$ in the control strips and by $10 \%$ in the SCT strips: thus, the active contractile force would be underestimated to a greater extent in SCT strips.

One test of contractility comprised the use of an inotropic agent targeted to detrusor muscle, namely the TRPV4 agonist GSK1016790A; however, similar observations were made with the muscarinic agonist carbachol. GSK1016790A evoked muscle contracture and a similar increase in the amplitude of spontaneous contractions in detrusor from both control and SCT bladders without altering their frequency. Given a significant reduction in interstitial cells in the SCT bladder wall [13], these data imply that spontaneous activity is not entirely interstitial cell-generated.

Overall, there is no evidence of contractile failure in the highly-compliant bladder and it is proposed that increased compliance is marked by altered matrix properties that dissipate muscle force to produce a compliant bladder that generates low pressures.

\section{Alterations to Extracellular Matrix in Bladder Hypertrophy}

Hypertrophy is associated with increased collagen deposition, at least in the early stages, as well as a decrease in the type I to type III ratio [24,25]. Collagen subtype changes may lead to changes in the passive mechanical properties described above because a decrease in the type I/III ratio is associated with reduced bladder compliance [25]. Conversely, the tendency of collagen III to form large coils may allow the bladder to hold large volumes more readily [26]. Altered collagen deposition and subtype composition are controlled by growth factors $[27,28]$, whose own release is up-regulated in the hypertrophic bladder [29]. Further studies are required to measure the changes in collagen distribution and subtype composition in the underactive bladder.

In conclusion, a model of detrusor underactivity associated with low bladder compliance was generated using spinal cord-transected rats. There was no evidence of impaired detrusor contractility and an overactive phenotype. However, reduced passive bladder wall stiffness and an enhanced rate of stress relaxation lead to the conclusion that changes to the tissue matrix will result in a low-pressure bladder. This implies that contractile agonists may be effective for improving bladder function and that long-term correction may be achieved by reversing the changes to passive bladder wall mechanical function.

\section{Acknowledgements}

John S. Young is funded by an AgeUK Research Fellowship. This work was funded by an EU FP7 grant 'INComb' (http://www.incomb.eu). The authors thank Dr R. J. Young (Department of Physics, University of Lancaster, UK) for development of the software analysing the frequency of spontaneous contractions.

\section{Conflict of Interest}

Gordon McMurray is an Employee of Pfizer and Christopher H. Fry is on the Advisory Board of Eli Lilly.

\section{References}

1 Abrams P, Cardozo L, Fall $M$ et al. The standardisation of terminology of lower urinary tract function: report from the Standardisation Sub-committee of the International Continence Society. Neurourol Urodyn 2002; 21: 167-78

2 Resnick NM, Yalla SV. Detrusor hyperactivity with impaired contractile function. an unrecognized but common cause of incontinence in elderly patients. JAMA 1987; 257: 3076-81

3 Cucchi A, Quaglini S, Rovereto B. Proposal for a urodynamic redefinition of detrusor underactivity. J Urol 2009; 181: 225-9

4 Taylor JA, Kuchel GA. Detrusor underactivity: clinical features and pathogenesis of an underdiagnosed geriatric condition. J Am Geriatr Soc 2006; 54: 1920-32

5 Krishnamoorthy S, Kekre NS. Detrusor underactivity: to tone or not to tone the bladder? Indian J Urol 2009; 25: 407-8

6 Fry $\mathrm{CH}$, Bayliss M, Young JS, Hussain M. Influence of age and bladder dysfunction on the contractile properties of isolated human detrusor smooth muscle. BJU Int 2011; 108: E91-6

7 Griffiths DJ, van Mastrigt R. The routine assessment of detrusor contraction strength. Neurourol Urodyn 1985; 4: 77-87

8 Griffiths DJ. Assessment of detrusor contraction strength or contractility. Neurourol Urodyn 1991; 10: $1-18$

9 Schäfer W. Analysis of bladder outlet function with the linearised passive urethral resistance relation, linPURR, and a disease-specific approach for grading obstruction: from complex to simple. World J Urol 1995; 13: 47-58 
10 Griffiths DJ. Detrusor contractility: order out of chaos. Scand J Urol Nephrol 2004; 215 (Suppl): 93-100

11 Sullivan MP, Yalla SV, DuBeau C, Cravalho EG. Continuous outlet occlusion test to determine detrusor contractile performance. J Urol 1995; 154: 1834-40

12 Tan TL, Bergmann MA, Griffiths D, Resnick NM. Stop test or pressure-flow study? Measuring detrusor contractility in older females. Neurourol Urodyn 2004; 23: $184-9$

13 Johnston L, Cunningham RM, Young JS et al. Altered distribution of interstitial cells and innervation in the rat urinary bladder following spinal cord injury. J Cell Mol Med 2011; [Epub ahead of print] doi: 10.1111/j.1582-4934.2011.01410.x

14 Thiruchelvam N, Wu C, David A, Woolf AS, Cuckow PM, Fry CH. Neurotransmission and viscoelasticity in the ovine fetal bladder after in utero bladder outflow obstruction. Am J Physiol 2003; 284: R1296-305

15 Thorneloe KS, Sulpizio AC, Lin Z et al. N-((1S)$1-\{[4-((2 S)-2-\{[(2,4-$ dichlorophenyl)sulfonyl $]$ amino $\}-$ 3-hydroxypropanoyl)-1-piperazinyl]carbonyl\}-3methylbutyl)-1-benzothiophene-2-carboxamide (GSK1016790A), a novel and potent transient receptor potential vanilloid 4 channel agonist induces urinary bladder contraction and hyperactivity: part I. J Pharmacol Exp Ther 2008; 326: 432-42

16 Ikeda Y, Kanai A. Urotheliogenic modulation of intrinsic activity in spinal cord-transected rat bladders: role of mucosal muscarinic receptors. Am J Physiol 2008; 295: F454-61

17 Resnick NM, Yalla SV, Laurino E. The pathophysiology of urinary incontinence among institutionalized elderly persons. N Engl J Med 1989; 320: $1-7$

18 Smith PP. Aging and the underactive detrusor: a failure of activity or activation? Neurourol Urodyn 2010; 29: 408-12

19 Pikov V, Wrathall JR. Altered glutamate receptor function during recovery of bladder detrusor-external urethral sphincter coordination in a rat model of spinal cord injury. J Pharmacol Exp Ther 2002; 300: 421-7

20 German K, Bedwani J, Davies J, Brading AF, Stephenson TP. Physiological and morphometric studies into the pathophysiology of detrusor hyperreflexia in neuropathic patients. J Urol 1995; 153: 1678-83

21 Drake MJ, Hedlund P, Mills IW et al. Structural and functional denervation of human detrusor after spinal cord injury. Lab Invest 2000; 80: 1491-9

22 Steers WD, Mackway Gerardi AM, Ciambotti J, de Groat WC. Alterations in neural pathways to the urinary bladder of the rat in response to STZ-induced diabetes. J Auton Nerv Syst 1994; 47: 83-94

23 Wyndaele JJ, Gammie A, Bruschini H et al. Bladder compliance what does it represent: can we measure it, and is it clinically relevant? Neurourol Urodyn 2011; 30: 714-22

24 Kim JC, Yoon JY, Seo SI, Hwang TK, Park YH. Effects of partial bladder outlet obstruction and its relief on types I and III collagen and detrusor contractility in the rat. Neurourol Urodyn 2000; 19: 29-42

25 Deveaud CM, Macarak EJ, Kucich U, Ewalt DH, Abrams WR, Howard PS. Molecular analysis of collagens in bladder fibrosis. J Urol 1998; 160: 1518-27

26 Chang SL, Howard PS, Koo HP, Macarak EJ. Role of type III collagen in bladder filling. Neurourol Urodyn 1998; 17: 135-45

27 Imamura M, Kanematsu A, Yamamoto S et al. Basic fibroblast growth factor modulates proliferation and collagen expression in urinary bladder smooth muscle cells. Am J Physiol 2007; 293: F1007-17

28 Chung CW, Zhang QL, Qiao LY. Endogenous nerve growth factor regulates collagen expression and bladder hypertrophy through Akt and MAPK pathways during cystitis. J Biol Chem 2010; 285: 4206-12

29 Yamaguchi O. Response of bladder smooth muscle cells to obstruction: signal transduction and the role of mechanosensors. Urology 2004; 63 (Suppl 1): 11-6

Correspondence: Christopher H. Fry, Institute of Biosciences and Medicine, University of Surrey, Guildford GU2 7XH, UK.

e-mail: c.h.fry@surrey.ac.uk

Abbreviations: DU, detrusor underactivity; SCT, spinal cord transection. 